\title{
A New Prospect of Additivity in Bankruptcy Problems*
}

\author{
José Alcalde ${ }^{\dagger}$, María del Carmen Marco-Gil ${ }^{\ddagger}$ and José A. Silva ${ }^{\S}$ \\ $\dagger$ IUDESP, University of Alicante, E-03071 Alicante, Spain. (e-mail: jose.alcalde@ua.es) \\ ¥ Dep. de Economía, Polytechnic University of Cartagena, Paseo Alfonso XIII, 50, 30203 Carta- \\ gena, Murcia, Spain. (e-mail: Carmen.Marco@upct.es) \\ $\S$ IUDESP and Dep. Fonaments de l'Anàlisi Econòmica, University of Alicante, E-03071 Ali- \\ cante, Spain. (e-mail: alasur@merlin.fae.ua.es)
}

\begin{abstract}
This paper explores additivity-like properties fulfiled by bankruptcy rules. Our main result is that the unique rule satisfying an adittivity-like property is the Minimal Overlap proposed by O'Neill. We also propose some conections relative to additivity properties in close frameworks as bargaining rules.
\end{abstract}

Keywords: Bankruptcy problems; Additivity; Minimal Overlap Rule JEL Classification Numbers: C71, D63, D71.

\footnotetext{
*This work is partially supported by the Institut Valencià d'Investigacions Econòmiques and the Spanish Ministerio de Educación y Ciencia under projects SEJ2007-62656 (Alcalde) and SEJ2007-64649 (Marco-Gil and Silva). Marco-Gil also acknowledges support by the Fundación Séneca of the Agencia de Ciencia y Tecnología of the Murcian Region, under project 05838/PHCS/07 (Programa de Generación Cientìfico de Excelencia).
} 


\section{Introduction}

The analysis of bankruptcy problems can be considered as a simple and robust tool to model how agents should be rationed. The idea behind these problems is that each agent in a group asks for a quantity of a perfectly divisible good; and they collectively claim that the available quantity is not enough to satisfy their demands.

The literature proposes two ways to approach how bankruptcy problems might be solved. The first one, namely normative, comes from an axiomatic analysis of rules that propose particular solutions. The paper by Thomson [16] proposes a nice overview of the main results following this approach. The second one is based on an interpretation of bankruptcy problems as (transferable utility) cooperative games, TU-games henceforth. This formulation, introduced by O'Neill [10], has been employed to justify the employ of the Random Arrival Rule due to its relationship with the Shapley value of the associated TU-game; or the use of the Talmudic rule because it coincides with the Nucleolus of the related TU-game (Aumann and Mashler [3]).

This paper proposes an approach to bankruptcy problems following its cooperative game-theoretical conception, and proposes a new characterization for the Minimal Overlap Solution (O'Neill [10]) by using a particular form of additivity.

Additivity has been employed as a natural property to be satisfied by solutions for TU-Games. For instance, in most of the characterizations provided in the literature for the Shapley value (Shapley [15]), additivity plays a central role. Moreover, in some problems (that can be) modeled as TU-games, additivity has also played a central role. In this sense we could mention that, for cost-sharing problems, additivity is also a central property to characterize the proportional rule; or in bargaining problems, Perles and Mashler [13] also employ additivity to characterize their solution.

Following the natural interpretation, by additivity we means that, given two bankruptcy problems the sum of their solutions should coincide with the solution for the bankruptcy problem generated by aggregating the two initial ones. In this sense, we should mention that, this natural way to define additivity is incompatible with some nondemanding properties, as Bergantiños and Méndez-Naya [4] pointed out. The reason is very simple and is based in the following observation. Given a bankruptcy problem, there is a unique TU-game associated to it; but the same TU-game can be generated by several bankruptcy problems. Thus, the fact that there is no bijection between bankruptcy problems and (a family) of TU-games can be employed to show that additivity, in its most natural conception, is a very demanding property. This is why in this paper we deal with a particular form of additivity. The idea behind this partial additivity comes from the interpretation above. Given that there is no bijection between bankruptcy problems and TU-games, is there possible to identify a family of bankruptcy problems where this relationship (with TU-games) holds? If we are able to identify such a subclass, it should be possible to guarantee the existence of additive bankruptcy rules 
on such a subclass. This is the aim of this paper.

The rest of the paper is organized as follows. In Section 2 we model bankruptcy problems and presents some bankruptcy solutions that will be used subsequently. Section 3 presents the interpretation of bankruptcy problems in terms of TU games given by O'Neill. Section 4 introduces bargaining problems and formalizes the relationship between them and bankruptcy problems. Section 5 is devoted to present, in all contexts previously considered, the concept of Additivity and some related results. Section 6 presents our main result, an axiomatic characterization of the Minimal Overlap bankruptcy rule based on a partial additivity property. Section 7 summarizes our main conclusions. Finally, the technical proofs are relegated to the Appendices.

\section{Bankruptcy Problems}

A Bankruptcy Problem can be formally described by a vector $(E, c) \in \mathbb{R}_{++} \times \mathbb{R}_{+}^{n}$ such that

$$
E \leq \sum_{i=1}^{n} c_{i}
$$

$E$ is known as the endowment, and represents the quantity of the perfectly divisible good that should be distributed among the agents in $N=\{1, \ldots, i, \ldots, n\}$, also called creditors. Each agent $i \in N$ has a claim $c_{i}$ on the endowment. Condition (2.1) reflects that agents' rights could be incompatible and hence, the endowment should be rationed.

Let $\mathcal{C}$ denote the set of all bankruptcy problems,

$$
\mathcal{C}=\left\{(E, c) \in \mathbb{R}_{++} \times \mathbb{R}_{+}^{n}: E \leq \sum_{i=1}^{n} c_{i}\right\} .
$$

For notational convenience, we will denote by $\mathcal{C}_{E}$ the set of problems in which there is some creditor demanding, at least, the total endowment,

$$
\mathcal{C}_{E}=\left\{(E, c) \in \mathcal{C}: E \leq \max _{i}\left\{c_{i}\right\}\right\}
$$

and, for any subclass of bankruptcy problems $\mathcal{C}_{*} \subseteq \mathcal{C}$, we will denote by $\mathcal{C}_{*}^{O}$ the set of problems in such a subclass with increasingly ordered claims,

$$
\mathcal{C}_{*}^{O}=\left\{(E, c) \in \mathcal{C}_{*}: c_{i} \leq c_{j} \text { for } i<j\right\} .
$$

Definition 2.1. A Bankruptcy rule is a function $\varphi: \mathcal{C} \rightarrow \mathbb{R}_{+}^{n}$, such that for each bankruptcy problem $C=(E, c) \in \mathcal{C}$,

(a) $\sum_{i \in N} \varphi_{i}(E, c)=E$, (efficiency) and 
(b) $0 \leq \varphi_{i}(E, c) \leq c_{i}$ for each creditor $i$, (non-negativity and claims boundedness).

Next, we introduce the Minimal Overlap, the Random Arrival and the Contested Garment bankruptcy rules, that will be used later. The formalism in their definitions depends only on subsequent needs.

Informally speaking, the Minimal Overlap bankruptcy rule, introduced by O'Neill [10], chooses the awards vector that minimizes the 'extent of conflict' over each available unit. Let us observe that this solution can be considered a generalization of the $I b n$ Ezra's bankruptcy rule ${ }^{1}$, which is only defined on $\mathcal{C}_{E}$.

We present the definition of the Minimal Overlap bankruptcy rule gathered in Alcalde, Marco and Silva [2], which is based in the result of Chun and Thomson [6].

Definition 2.2. The Minimal Overlap bankruptcy rule is the function

$$
\varphi^{m o}: \mathcal{C}^{O} \rightarrow \mathbb{R}_{+}^{n}
$$

which associates, to any bankruptcy problem $(E, c)$ in $\mathcal{C}_{O}$, and creditor $i$, the share of the endowment,

$$
\varphi_{i}^{m o}(E, c)=\sum_{j=1}^{i} \frac{\min \left\{c_{j}, t\right\}-\min \left\{c_{j-1}, t\right\}}{n-j+1}+\max \left\{c_{i}-t, 0\right\},
$$

where $c_{0}=0$, and

(a) $t=E$ if $E \leq c_{n}$, or

(b) otherwise, $t$ is the unique solution for the equation

$$
\sum_{k=1}^{n} \max \left\{c_{k}-t, 0\right\}=E-t
$$

Remark 1. Just to extend Definition 2.2 to $\mathcal{C}$, let us note that, for any bankruptcy $(E, c)$ in $\mathcal{C} \backslash \mathcal{C}^{O}$ there is a permutation ${ }^{2} \pi$ such that $(E, \pi(c))$ is in $\mathcal{C}^{O}$. Hence we can compute

$$
\varphi^{m o}(E, c)=\pi^{-1}\left[\varphi^{m o}(E, \pi(c))\right] .
$$

\footnotetext{
${ }^{1}$ Anoter way of extending the Ibn Ezra's proposal, called the Generalized Ibn Ezra banruptcy rule, is provided by Alcalde, Marco and Silva [1]. They apply recursivity and impose that the general principle from which it is inspired remains fixed.

${ }^{2}$ Given a set of agents $N$, we denote by $\Pi^{N}$ the class of bijections from $N$ into itself, and by $\pi$ an element in $\Pi^{N}$. Throughout the rest of the paper, and abusing notation, $\pi(c)$ will denote the claims vector obtained by applying permutation $\pi$ to its components, i.e. the $i$-th component for $\pi(c)$ is $c_{j}$ whenever $j=\pi(i)$.
} 
To introduce the Random Arrival bankruptcy rule, imagine creditors arriving one at a time, and compensate them fully until money runs out. The resulting awards vector of course depends on the order in which creditors arrive. To remove the unfairness associated with a particular order, take the arithmetic average over all orders of arrival of the awards vectors calculated in this way, O'Neill [10].

Definition 2.3. The Random Arrival bankruptcy rule is the function

$$
\varphi^{r a}: \mathcal{C} \rightarrow \mathbb{R}_{+}^{n}
$$

which associates, to each bankruptcy problem $(E, c)$ in $\mathcal{C}$, and each creditor $i \in N$, the share of the endowment

$$
\varphi_{i}^{r a}(E, c)=\frac{1}{n !} \sum_{\pi \in \Pi^{N}} \min \left\{c_{i}, \max \left\{\left\{E-\sum_{j \in N, \pi(j)<\pi(i)} c_{j}, 0\right\}\right\} .\right.
$$

The rationale of the Contested Garment bankruptcy rule, defined only for twocreditors problems in the Babylonian Talmud (Baba Metzia, 2a), is provided by Aumann and Maschler [3] as follows. Suppose that the endowment, $E$, is allocated in two stages. In the first stage, each creditor $i$ gets whatever the other concedes, that is, he gets $\max \left\{E-c_{j}, 0\right\}$, leaving the rest for the second stage, $E-\sum_{i=1,2} \max \left\{E-c_{j}, 0\right\}$. In this last stage, the remainder, the part that is truly contested, is divided equally between the claimants. Equal division in this stage makes sense since both claims becomes equal after being revised down by the amounts received in the first stage, and truncated by the amount that remains available.

Definition 2.4. The Contested Garment bankruptcy rule for two-creditors problem is the function $\varphi^{c d}$ which associates, to each 2-agent bankruptcy problem the share of the endowment

$$
\begin{aligned}
\varphi_{1}^{c d}(E, c) & =\frac{E+\max \left\{E-c_{1}, 0\right\}-\max \left\{E-c_{2}, 0\right\}}{2} \\
\varphi_{2}^{c d}(E, c) & =\frac{E+\max \left\{E-c_{2}, 0\right\}-\max \left\{E-c_{1}, 0\right\}}{2}
\end{aligned}
$$

The fact that for two-creditors problems, both the Minimal Overlap and the Random Arrival bankruptcy rules coincide with the Contested Garment bankruptcy rule is well known, however, these bankruptcy rules are not the only ones generalizing the Contested Garment bankruptcy rule to n-creditors bankruptcy problems, but also the Talmud and the Adjusted Proportional bankruptcy rules. The Talmud bankruptcy rule, 
was introduced in a very nice paper by Aumann and Maschler [3], on the basis of Consistency. And the Adjusted Proportional bankruptcy rule, introduced by Curiel, Maschler and Tijs [7], being its ground to divide the remainder, once each creditor has got the amount corresponding to his minimal right, $m_{i}=\max \left\{E-\sum_{j \neq i} c_{j}, 0\right\}$, in proportion to the part claimed by the creditors, of such a residual endowment, according to their outstanding claims.

\section{Bankruptcy Problems and TU Games}

The first general approach for the multiperson coalitional games was proposed by von Neumann and Morgenstern [19]. Next we present the basic concept of their model.

A TU game involving a set of agents $N$ can be described as a function $V$ associating a real number to each subset of agents, or coalition, $S$ contained in $N$. Formally, a TU game is a pair $(N, V)$, where $V: 2^{N} \rightarrow \mathbb{R}$. For each coalition $S \subseteq N, V(S)$ is commonly called its worth and denotes the quantity that agents in $\mathrm{S}$ can guarantee to themselves if they cooperate. So that, it is assumed that $V(\varnothing)=0$. It is often assumed that $(N, V)$ is Superadditive, that is, for any pair of coalitions $S, T \subset N$ such that $S \cap T=\varnothing$, $V(S \cup T) \geq V(S)+V(T)$, so that there is incentive for the grand coalition $N$ forms.

A single-valued solution for TU games, a TU value, is described by a function selecting, for each TU game, a share of the worth of the grand coalition among the agents in such a game. Let $\mathcal{G}$ be a family of TU games referred to a fixed set of agents, say $N$.

Definition 3.1. A TU-value is a function $\gamma: \mathcal{G} \rightarrow \mathbb{R}^{n}$, such that for each $T U$ game $G=(N, V) \in \mathcal{G}$,

$$
\sum_{i \in N} \gamma_{i}(N, V)=V(N)
$$

Let us remark that Condition 3.1 incorporates both Feasibility and Pareto Optimality. The requirement of Feasibility says that the members of the grand coalition can actually achieve the selected share, Pareto Optimality imposes that they cannot achieve more. Usually, Individual Rationality is also required, what means that no individual can achieve more than the amount allocated to him as a payoff, for each $i \in N$, $\gamma_{i}(N, V) \geq V(\{i\})$.

Next, we present the Shapley value, whose interpretation, given by Shapley [15] is the following: 'The players in $N$ agree to play the game $V$ in a grand coalition, formed in the following way: (i) Starting with a single member, the coalition adds one player at a time until everybody has been admitted. (ii) The order in which the players are to join is determined by chance, with all arrangements equally probable. (iii) Each player, on his admission, demands and is promised the amount which his adherence contributes to 
the value of the coalition (as determined by the function $V$ ). The grand coalition then plays the game 'efficiently' so as to obtain $V(N)$, exactly enough to meet all promises.'

Definition 3.2. The Shapley TU value is the function

$$
\gamma^{s h}: \mathcal{G} \rightarrow \mathbb{R}^{n}
$$

which associates, to each $T U$ game $(N, V)$ in $\mathcal{G}$, and each creditor $i \in N$, the share of the worth of the grand coalition

$$
\gamma_{i}^{s h}(N, V)=\sum_{S \subseteq N \backslash\{i\}} \frac{|S| !(n-|S|-1) !}{n !}(V(S U\{i\})-V(S)) .
$$

From now on, given a TU game $(N, V)$, for each agent $i \in N$ and each coalition $S \subset N$, we call the Marginal Contribution of agent $i$ to coalition $S$, to the amount which his adherence contributes to the value of the coalition, that is $V(S U\{i\})-V(S)$.

O'Neill [10] proposed an interpretation of bankruptcy problems as TU games. His advise was to associate to each coalition the part of the endowment, if any, that remains after paying the debts that the bankrupted contracted with all his creditors outside this coalition. Given a bankruptcy problem $C=(E, c)$, we denote by $\left(N, V_{C}\right)$ the TU game that it induces. $\mathcal{G}_{C}$ will denote the set of TU-bankruptcy games.

Definition 3.3. Let $C=(E, c)$ be a bankruptcy problem in $\mathcal{C}$, the $T U$ game induced by $C$, called TU-bankruptcy game, is the pair $\left(N, V_{C}\right)$, where the function $V_{C}: 2^{N} \rightarrow \mathbb{R}$ associates, to each coalition $S \subseteq N$, the real value

$$
V_{C}(S)=\max \left\{E-\sum_{i \in N \backslash S} c_{i}, 0\right\} .
$$

The previous link, between bankruptcy problems and TU games, could allow us to translate some of the results and properties relative to values for $\mathrm{TU}$ games into bankruptcy theory and vice versa. Before analyzing this possibility, let us introduce the properties: Respect of Minimal Rights, Minimal Rights First, Invariance under Claims Truncation and Translation Invariance. ${ }^{3}$

The first property is a requirement placing a lower bound on awards, that corresponding to the minimal right of each creditor.

\footnotetext{
${ }^{3}$ Since, through the paper, we are to introduce properties relative to bankruptcy problems, bargaining situations and TU-games, we follow the convention of distinguishing C-properties, B-properties and TUproperties respectively just to clarify the framework in which they are employed.
} 
Property 1. C-RESPECT OF MINIMAL RIGHTS.

For each $(E, c) \in \mathcal{C}$, and each $i \in N, \varphi_{i}(E, c) \geq m_{i}(E, c)=\max \left\{E-\sum_{N \backslash\{i\}} c_{j}, 0\right\}$.

This property is a consequence of efficiency, non-negativity and claims boundedness together. So that, any bankruptcy rule (See Definition 2.1) satisfies Respect of Minimal Rights.

It can be checked that the next two properties that we present, are satisfied by the Contested Garment, the Random Arrival and the Minimal Overlap bankruptcy rules.

The first one requires that the awards vector is equivalently obtained: (i) directly, or (ii) by first assigning to each agent his minimal right, adjusting claims down by these amounts, and finally, applying the bankruptcy rule to divide the remainder.

Property 2. C-MINIMAL RIGHTS FIRST.

For each $(E, c) \in \mathcal{C}, \varphi(E, c)=m(E, c)+\varphi\left(E-\sum_{i \in N} m_{i}(E, c), c-m(E, c)\right)$.

The next requirement is that the part of a claim that is above the amount to divide should be ignored.

\section{Property 3. C-INVARIANCE UNDER CLAIMS TRUNCATION.}

For each $(E, c) \in \mathcal{C}, \varphi(E, c)=\varphi\left(E, c^{E}\right)$, where $c_{i}^{E}$ is interpreted as the part of $E$ claimed by creditor $i$, given that he cannot claim more than there is, $c_{i}^{E}=\min \left\{c_{i}, E\right\}$.

The following condition imposes that origin changes do no affect the recommended share in a TU game.

Property 4. TU-TRANSLATION INVARIANCE.

For each $(N, V) \in \mathcal{G}$, and for any n-dimensional vector $a=\left(a_{1}, \ldots, a_{n}\right), \gamma(N, W)=$ $\gamma(N, V)-a$, where for each $S \subseteq N, W(S)=V(S)-\sum_{i \in S} a_{i}$.

This property is standard in TU games, and most of the solutions proposed in this context satisfy it. In fact,it is usual the analysis of TU-games from its 0-normalization, that is the origin change in which the worth of any individual coalition is zero.

Now, in order to analyze the connections between bankruptcy problems and TU games, two natural questions arise when considering solution concepts.

First, let $\gamma$ be a TU value, could we interpret $\gamma$ as a bankruptcy rule just identifying for each $C=(E, c), \varphi(C)$ with $\gamma\left(N, V_{C}\right)$ ? It can be checked straightforwardly that a TU value $\gamma$ is a bankruptcy rule if and only if $\gamma$ satisfies TU-Individual rationality.

Second, let $\varphi$ be bankruptcy rule then, is there a TU value $\gamma$ such that for any bankruptcy problem $C=(E, c), \varphi(C)=\gamma\left(N, V_{C}\right)$ ? Curiel, Maschler and Tijs.[7] showed 
that a bankruptcy rule $\varphi$ is a value for TU-bankruptcy games if and only if $\varphi$ satisfies C-Invariance under Claims Truncation.

If for each bankruptcy problem, the recommendation made by a given bankruptcy rule coincides with the recommendation made by a given TU-value when applied to the induced TU-bankruptcy game, we say that 'The bankruptcy rule corresponds to the TUvalue'. Relative to the bankruptcy rules considered in this work, O'Neill [10] proposed the following result.

Theorem 3.4. The Random Arrival bankruptcy rule corresponds to the Shapley TUvalue.

In a similar way, it could be defined a property for bankruptcy problems from the straight application of some appealing or natural requirement for TU games. But in this case, the question would be the following, metaphorically speaking. Is the 'essence' of a property unchanged in its travel from TU games to bankruptcy problems? If 'essence' is understood as meaning and reasonableness, several situations can be found. Next we provide some examples. It is obvious that TU-Pareto Optimality keeps its full 'essence'. TU-Individual Rationality corresponds to C-Respect of Minimal Rights, therefore its meaning changes (let us note that the translation of the interpretation of TU-Individual Rationality would be C-non-negativity, meanwhile C-Respect of Minimal rights is a consequence of efficiency, non-negativity and claim boundedness together), but both properties would have, from our point view, similar rank in a scale measuring reasonableness. TU-Translation Invariance losses absolutely its 'essence', although there has been some attempt to retrieve it by restricting its translation. To this regard, CRespect of Minimal Rights, whose interpretation comes from being a particular kind of composition, can be seen as the application of TU-Translation Invariance when allowing only origin changes by using the Minimal Rights vector. So that meaning has been changed but some reasonableness has been got.

\section{Bankruptcy Problems and Bargaining Games}

The axiomatic theory of bargaining originated in a paper by Nash [9], where he introduced an idealized representation of the bargaining problem and developed a methodology to solve it. The formal model is as follows.

A Bargaining game is a pair $(B, d)$ where $B \subset \mathbb{R}^{n}$ is a compact and convex set, $d \in B$ and there exists $x \in B$ such that $x_{i} \geq d_{i}, i=1, . ., n$ with some strict inequality. $(B, d)$ represents a situation where a set of individuals, denoted by $N$, bargain over a set of possible agreements. $B$ is the set of all feasible utility allocations that may be reached by means of an agreement. If there is no agreement, each individual $i$ gets $d_{i}$, the utility corresponding to the disagreement point $d$. From now on we deal with the 
class of bargaining games where the disagreement point is zero, $d=0$, and we omit $d$ in the notation altogether. Let us denote by $\mathcal{B}$ this class of bargaining problems.

Definition 4.1. A Bargaining solution is a function $\beta: \mathcal{B} \rightarrow \mathbb{R}^{n}$, that assigns each $B \in \mathcal{B}$ a unique element of $B$, to be interpreted as a compromise among agents or, alternatively, as the recommendation that an impartial arbitrator would make.

Although the Nash bargaining solution was regarded as 'the solution' in this context until the mid-seventies, from then this model expanded in several directions. (See the surveys by Thomson [17], [18].)

In this context, and related to our aim in this paper about additivity, it should be mentioned the Perles-Maschler [13] bargaining solution, originally introduced for two-person bargaining problems. In order to present it, we need additional notation. Let $\partial B=\left\{x \in B: \nexists x^{\prime} \in B\right.$, with $\left.x^{\prime}>x\right\}$ be the undominated boundary of $B$. Let $P O(B)=\left\{x \in B: \nexists x^{\prime} \in B\right.$, with $\left.x^{\prime} \geq x\right\}$ be the strictly undominated boundary of $B$. And let $D^{* i}(B)$ be the point of $P O(B)$ of maximal $i$-th coordinate.

The Perles-Maschler bargaining solution is based on a process of balanced concessions: agents work their way from their preferred alternatives, the shares provided by $D^{* i}(B)$ for each one, to a final position by moving from compromise to compromise. According to this bargaining solution, balanced is interpreted as follows: the area of the set of alternatives that each agent renounces in each movement is the same.

Definition 4.2. The Perles-Maschler bargaining solution for two-creditors problem with polygonal $\partial B, \mathcal{B}_{2 P}=\{B \in \mathcal{B}:|N|=2$ and $\partial B$ is polygonal $\}$, is the function

$$
\beta^{p m}: \mathcal{B}_{2} \rightarrow \mathbb{R}^{n}
$$

which associates, to each bargaining game $B \in \mathcal{B}_{2 P}$, and each player $i \in N$, the share of the endowment which is the common limit point of the sequences $\left\{x^{t}\right\},\left\{y^{t}\right\}$, defined by: $x^{o}=D^{* 1}(B), y^{o}=D^{* 2}(B)$; for each $t \in N, x^{t}, y^{t} \in P O(B)$ are such that $x_{1}^{t} \geq y_{1}^{t}$, the segments $\left[x^{t-1}, x^{t}\right],\left[y^{t-1}, y^{t}\right]$ are contained in $P O(B)$ and the products $\left(x_{1}^{t-1}-x_{1}^{t}\right)\left(x_{2}^{t}-x_{2}^{t-1}\right)$ and $\left(y_{1}^{t}-y_{1}^{t-1}\right)\left(y_{2}^{t-1}-y_{2}^{t}\right)$ are equal and maximal.

If $\partial B$ is not polygonal, $\beta^{p m}$ is defined by approximating $B$ by a sequence of polygonal problems and taking the limit of the associated solution outcomes.

Next, we present a natural way of linking bankruptcy problems and bargaining games, which is due to Dagan and Volij [8]. Let us note that one could argue that too much information is lost in the passage from bankruptcy problems to bargaining games. 
Definition 4.3. Let $C=(E, c)$ be a bankruptcy problem in $\mathcal{C}$, the 0-Bankrupcy game induced by $C$ is denoted by $B(C)=\left\{x \in \mathbb{R}_{+}^{n}: 0 \leq x \leq c, \sum_{i \in N} x_{i} \leq E\right\}$.

Previous definition provides the key to travel from bankruptcy problems to bargaining games. It means that the individuals are bargaining over all the possible divisions of the endowment $E$ that give each one no more than his claim and no less than zero, and that if they fail to reach an agreement, no one gets anything. In order to get something, they need to reach an unanimous agreement.

In this context, the unique restriction for guaranteeing that a bargaining solution is a bankruptcy rule is Pareto Optimality. And all bankruptcy rules are 0-bankruptcy game solutions.

Similarly to the above Section, we will say that 'a bankruptcy rule corresponds to a bargaining solution if for each bankruptcy problem, the recommendation made by a given bankruptcy rule coincides with the recommendation made by a given bargaining solution when applied to the induced 0-bankruptcy game. Relative to the bankruptcy rules considered in this work, Dagan and Volij [8] proposed the following result.

Theorem 4.4. For two-person problems the Contested Garment bankruptcy rule corresponds to the Perles-Maschler bargaining solution.

Dagan and Volij [8] referred to the previous theorem as follows. 'This result is quite surprising, since we could not get a bankruptcy rule satisfying C-Minimal Rights First from the application of the other bargaining solutions to the associated 0-bankruptcy game. It would be very interesting to check what bankruptcy rule corresponds with the generalization of the Perles-Maschler bargaining solution to n-person bargaining problems, when and if such a generalization appears.'

\section{Some Results Related to Additivity}

In this Section we present formally the idea of Additivity for TU games and bargaining games and present the axiomatic characterizations of solutions involving such a property in both contexts. Moreover, we point out the difficulties when translating Additivity from both models to bankruptcy problems, what will lay the foundations to go into Additivity for bankruptcy problems in depth.

Two different interpretations have been traditionally used to justify Additivity of a solution concept. The first one is based on risk neutrality: players facing a lottery of two games do not distinguish between the value of the expected game and the expected value

\footnotetext{
${ }^{4}$ An alternative specification of this conection, also due to Dagan and Volij [8], can be made by identifying the disagreement point with the minimal rights vector.
} 
of the games. The other one comes from imposing to be irrelevant that players facing in two remote games evaluate their expectations either in both games independently or by establishing a package deal.

Let us go on with the concept of Additivity for TU games.

Definition 5.1. Let $\gamma$ a solution for bargaining games. We say that $\gamma$ satisfies $T U$ Additivity if for all pairs of $T U$ games in $\mathcal{G}, G=(N, V)$ and $G^{\prime}=\left(N, V^{\prime}\right)$,

$$
\gamma\left(N, V+V^{\prime}\right) \geq \gamma(N, V)+\gamma\left(N, V^{\prime}\right),
$$

where the game $\left(V+V^{\prime}\right)$ is defined by $\left(V+V^{\prime}\right)(S)=V(S)+V^{\prime}(S)$ for all $S \subseteq N$.

This property was the main ground used by Shapley [15] to introduced his proposal for solving TU games. Next we present his axiomatic characterization for what we need the following two standard properties for TU games.

The first one requires the following notion of symmetry: players $i, j \in N$ are said to be symmetric with respect to game $(N, V)$ if they make the same marginal contribution to any coalition, that is, for each $S \subset N$ with $i, j \notin S, V(S U\{i\})=V(S U\{j\})$. The Symmetry axiom requires symmetric players to be paid equal shares.

Axiom 1. TU-SYMMETRY.

If players $i$ and $j$ are symmetric with respect to game $(N, V)$, then $\gamma_{i}(N, V)=$ $\gamma_{j}(N, V)$.

The second property requires that zero payoffs be assigned to players whose marginal contribution is null with respect to every coalition.

Axiom 2. $T U-D U M M Y$.

If $i$ is a dummy player, that is, $V(S U\{i\})-V(S)=0$ for every $S \subset N$, then $\gamma_{i}(N, V)=0$.

Theorem 5.2. [Shapley [15]]. The Shapley $T U$ value is the only $T U$ value on $\mathcal{G}$ satisfying TU-Symmetry, TU-Dummy and TU-Additivity.

Given that the Random Arrival bankruptcy rule corresponds to the Shapley TUvalue, it could be expected to identify the Random Arrival bankruptcy rule by means of the translation of TU-Additivity to bankruptcy problems, but the following example shows the difficulties of such a link.

Example 5.3. Let us consider $C=(4.5 ;(4,4,4))$ and $C^{*}=(15.5 ;(2,6,11))$. It can be checked that $V_{C}(\{2\})=V_{C}(\{3\})=0, V_{C^{*}}(\{2\})=2.5$ and $V_{C^{*}}(\{3\})=7.5$. Now, we have that $C+C^{*}=(20 ;(6,10,15)), V_{C+C^{*}}(\{2\})=0$ and $V_{C+C^{*}}(\{3\})=4$. But $\left(V_{C}+V_{C^{*}}\right)(\{2\})=2.5$ and $\left(V_{C}+V_{C^{*}}\right)(\{3\})=7.5$. Therefore, the $T U$ game $\left(N, V_{C}+V_{C^{*}}\right)$ does not correspond to the bankruptcy problem $C+C^{*}$. 
Next, we analyze the idea of additivity for Bargaining Games.

Definition 5.4. Let $\beta$ a solution for bargaining games. We say that $\beta$ satisfies $B$ Additivity if for all pairs of bargaining games in $\mathcal{B}, B$ and $B^{\prime}$,

$$
\beta\left(B+B^{\prime}\right) \geq \beta(B)+\beta\left(B^{\prime}\right),
$$

where $y \in\left(B+B^{\prime}\right)$ if and only if $\exists x \in B, x^{\prime} \in B^{\prime}$ such that $y=x+x^{\prime}$.

Perles and Maschler used three standard properties on bargaining games to identify, together with B-Additivity, their bargaining solution for two-agent problems.

The first property says that if the agents cannot be differentiated on the basis of the information contained in the mathematical description of $B$, then the solution should treat them the same.

Axiom 3. B-SYMMETRY.

If $B$ is invariant under all exchanges of agents, $\beta_{i}(B)=\beta_{j}(B)$ for all $i, j \in N$.

The second property requires that the solution should be independent of the following linear transformations. Let $\Lambda^{n}: \mathbb{R}^{n} \rightarrow \mathbb{R}^{n}$ be the class of independent person by person, positive, linear transformation with zero as independent term: $\lambda \in \Lambda^{n}$ if there is $b \in \mathbb{R}_{++}^{n}$ such that for all $x \in \mathbb{R}^{n}, \lambda(x)=\left(b_{1} x_{1}, \ldots, b_{n} x_{n}\right)$. Given $\lambda \in \Lambda^{n}$ and $B \subset \mathbb{R}^{n}$, $\lambda(B)=\left\{x^{\prime} \in \mathbb{R}^{n}: \exists x \in B\right.$ with $\left.x^{\prime}=\lambda(x)\right\}$.

Axiom 4. B-SCALE INVARIANCE.

For each $B \in \mathcal{B}$ and each $\lambda \in \Lambda^{n}, \lambda(\beta(B))=\beta(\lambda(B))$.

The last property considered requires that small changes in bargaining problems do not lead to wildly different solution outcomes.

Axiom 5. B-CONTINUITY.

For each sequence of bargaining problems, $\left\{B^{\nu}\right\}_{\nu=1, . ., n}$ with $B^{\nu} \in \mathcal{B}$ for all $\nu$, If $\lim _{\nu \rightarrow \infty} B^{\nu}=B$ in the Hausdorff topology, then $\lim _{\nu \rightarrow \infty} \beta\left(B^{\nu}\right)=\beta(B)$.

Finally, we will say that a bargaining problem $B \in \mathcal{B}$ is strictly comprehensive if the undominated boundary of $B, \partial B$, does not contain a segment parallel to an axis.

Theorem 5.5. [Perles and Maschler [13]]. For two-agent bargaining problems, the Perles-Maschler bargaining solution is the only bargaining solution on $\mathcal{B}$ satisfying $B$ Symmetry, B-Scale Invariance and B-Additivity, and to be B-Continuous on the subclass of $\mathcal{B}$ of strictly comprehensive bargaining problems. 
Now, taking into account that both the Random Arrival and the Minimal Overlap bankruptcy rules correspond to the Perles and Maschler bargaining solution, it could be expected to identify some of them by means of the translation of B-Additivity to bankruptcy problems. But in this case the basis of this expectations are weaker. On the one hand, Perles [12] showed that the axioms characterizing the Perles-Maschler bargaining solution are incompatible even for three-person unanimity bargaining games, what raised the problem of finding a satisfactory generalization of such a solution. In this line are the works of Peters [14], Calvo and Gutiérrez [5] and Pallaschke and Rosenmüller [11]. On the other hand, the following example shows that B-Additivity losses its meaning and reasonableness when translating to bankruptcy problems.

Example 5.6. Let us consider $C=(50 ;(20,60))$ and $C^{*}=(30 ;(40,40))$, then $C+$ $C^{*}=(80 ;(60,100))$. It can be checked that vector $(60,20) \in B\left(C+C^{*}\right)$ but $(60,20)$ $\notin\left(B(C)+B\left(C^{*}\right)\right)$. Therefore, the bargaining game $\left(B(C)+B\left(C^{*}\right)\right)$ does not correspond to the bankruptcy problem $C+C^{*}$.

Another possibility for requiring Additivity in bankruptcy problems, given the negative findings above presented, is to analyze the direct application of such a concept on the context at hand.

Definition 5.7. Let $\varphi$ a bankruptcy rule. We say that $\varphi$ satisfies $C$-Additivity if for all pairs of bankruptcy problems in $\mathcal{C}, C=(E, c)$ and $C^{\prime}=\left(E^{\prime}, c^{\prime}\right)$,

$$
\varphi\left(E+E^{\prime}, c+c^{\prime}\right) \geq \varphi(E, c)+\varphi\left(E^{\prime}, c^{\prime}\right)
$$

Unfortunately, it is known that there is no bankruptcy rule satisfying C-Additivity, fact that is gathered by Thomson [16]. The following example, due to Bergantiños and Méndez-Naya [4], provides this impossibility result.

Example 5.8. Let $\varphi$ a $C$-Additive bankruptcy rule and suppose that

$$
\varphi(10,(5,15))=(10-x, x) .
$$

If we take $y \neq x, 5 \leq y \leq 10$ by the $C$-Additivity of $\varphi$ we have that

$$
\varphi(10,(5,15))=\varphi(y,(0,15))+\varphi(10-y,(5,0))=(10-y, y)
$$

and

$$
(10-y, y) \neq(10-x, x) .
$$


At this point, let us notice that the fact that C-Additivity is a very strong requirement in bankruptcy problems is not very surprising, since the amount that a creditor gets in a bankruptcy problem is very sensitive to the relative position of her claim in front of both the total amount to be shared and the other creditors' claims and the property of C-Additivity allows great differences, in this sense, among the involved bankruptcy problems. To avoid this, Bergantiños and Méndez-Naya [4] analyze C-Additivity in a restricted domain of bankruptcy problems, those ones in which (i) the order of the creditors' claims is fixed and (ii) there is always at least one creditor claiming the total endowment. They identify the Ibn Ezra's bargaining solutions by means of C-Additivity in this context. But, from our viewpoint, although condition (i) could be quite suitable, the domain is really restrictive since bankruptcy problems fulfill condition (ii) are very unusual.

\section{Partial Additivity and the Minimal Overlap Rule}

This section deals with the possibility of finding a notion of partial additivity for that could be satisfied by some bankruptcy rules. To reach our objective we first impose two condition on the problems to be added. The first condition is that the position of any creditor is the same in both bankruptcy problems, when they are ranked on the basis of the claimed amounts; the second one is that the position of any creditor's claim, with regard to the total amount to be shared, does not reverse from a bankruptcy problem to the other one. Formally,

Property 5. FIXED RELATIVE POSITION CLAIMS ADDITIVITY.

For each pair of bankruptcy problems in $\mathcal{C}, C=(E, c)$ and $C^{\prime}=\left(E^{\prime}, c^{\prime}\right)$, such that

(i) for all $i, j \in N, i \neq j, c_{i} \leq c_{j} \Longleftrightarrow c_{i}^{\prime} \leq c_{j}^{\prime}$ and

(ii) for all $i \in N,\left(E-c_{i}\right)\left(E^{\prime}-c_{i}^{\prime}\right) \geq 0$,

$$
\varphi\left(E+E^{\prime}, c+c^{\prime}\right)=\varphi(E, c)+\varphi\left(E^{\prime}, c^{\prime}\right) .
$$

Let us note that in Example 5.8 the two conditions above, (i) and (ii), are not met. However, and unfortunately, the following example shows that there is no bankruptcy rule satisfying this kind of Additivity together with Anonymity, a generally accepted property demanding each agent's reward depends on the entire structure of the problem rather than her label (see Axiom 7 bellow, for a formal definition).

Example 6.1. Let us suppose that there is an Anonymous bankruptcy rule satisfying Fixed Relative Position Claims Additivity, say $\varphi$, then on the one hand

$$
\varphi_{1}(20,(6,10,11))=\varphi_{1}(14,(5,5,5))+\varphi_{1}(6,(1,5,6))=14 / 3=4+(2 / 3),
$$


and on the other hand

$$
\begin{aligned}
\varphi_{1}(20,(6,10,11)) & =\varphi_{1}(6,(4,4,4))+\varphi_{1}(14,(2,6,7)) \Longrightarrow \\
2 & \leq \varphi_{1}(20,(6,10,15)) \leq 4
\end{aligned}
$$

which obviously implies that $\varphi$ is not a bankruptcy rule.

Example 6.1 clarifies some central characteristic which affects decisively the endowment distribution in a bankruptcy problem and that, up to now, has not been considered when adding up them. Specifically, we refer to the existence of parts of the claims which 'are not on quarrel' in the following sense. Let us consider a given agent, $i \in N$. The following situations would guarantee that some part of agent $i$ 's claim is not on quarrel:

(a) Agent $i$ is the highest claimant and:

(i) There is nobody matching his claim, or

(ii) The endowment is greater than his claim.

(b) Agent i is not the highest claimant and:

(i) After as much as possible of the excess claims of all agents, with respect to agent $i$ 's claim, is reimbursed, the remainder endowment is greater that what agent $i$ 's claim.

Formally, given a bankruptcy problem $(E, c) \in \mathcal{C}$, and an agent $i \in N$, let $e_{j}^{i}(E, c)=$ $\max \left\{0,\left(c_{j}-c_{i}\right)\right\}$ be $j$ 's excess claim with respect to $i$ 's one (which is a positive number when $c_{j}>c_{i}$ and zero otherwise). Summing over $j$ for all $j \neq i$ we get the aggregate excess claim $\sum_{j \in N} e_{j}^{i}(E, c)$ relative to the $i$-th agent. Then

Definition 6.2. Given a bankruptcy problem in $\mathcal{C}, C=(E, c)$ We say that the $i$-th agent's claim is 'Partially Indisputable' in $(E, c)$ if either

(a) $\max _{j \in N \backslash\{i\}}\left\{c_{j}\right\}<\min \left\{c_{i}, E\right\}$ or

(b) $\max \left\{0, E-\sum_{j \in N \backslash\{i\}} e_{j}^{i}(E, c)\right\}>c_{i}$, whenever $\max _{j \in N \backslash\{i\}}\left\{c_{j}\right\} \geq c_{i}$.

The following 'partial' Additivity that we introduce, imposes that the quality of partial indisputableness of the creditors' claims does not change in the bankruptcy problems that will be added up. Otherwise we could find situations where any unit claimed by an agent would be disputed in the complete bankruptcy problem meanwhile some of them would not by regarding the problem separately. Formally, 
Axiom 6. Fixed Non-Quarrelled Claims Additivity

Let $\varphi$ a rule for bankruptcy problems. We say that $\varphi$ satisfies Fixed Non-Quarrelled Claims Additivity if for all pairs of bankruptcy problems in $\mathcal{C}, C=(E, c)$ and $C^{\prime}=$ $\left(E^{\prime}, c^{\prime}\right)$, such that

(a) for all $i \neq j, c_{i} \leq c_{j} \Longleftrightarrow c_{i}^{\prime} \leq c_{j}^{\prime}$,

(b) for all $i \in N,\left(E-c_{i}\right)\left(E^{\prime}-c_{i}^{\prime}\right) \geq 0$ and

(c) for all $i \in N, c_{i}$ is partially indisputable $\Longleftrightarrow c_{i}^{\prime}$ is partially indisputable,

$$
\varphi\left(E+E^{\prime}, c+c^{\prime}\right)=\varphi(E, c)+\varphi\left(E^{\prime}, c^{\prime}\right)
$$

Remark 2. To understand the extent of Fixed Non-Quarrelled Claims Additivity, it is worth noticing that in the subclass of bankruptcy problems $\mathcal{C}_{E}$ this property can be applied to any pair of problems in $\mathcal{C}, C=(E, c)$ and $C^{\prime}=\left(E^{\prime}, c^{\prime}\right)$, for which the order of the agents, on the basis of the amount claimed, is strictly preserved, that is we only need to demand that for all $i \neq j, c_{i}<c_{j} \Longleftrightarrow c_{i}^{\prime}<c_{j}^{\prime}$ and $c_{i}=c_{j} \Longleftrightarrow c_{i}^{\prime}=c_{j}^{\prime}$.

Our next result not only shows that some kind of Additivity can be fulfilled in the class of bankruptcy problems but also that Fixed Non-Quarrelled Claims Additivity is the distinctive property of the Minimal Overlap Rule. Before providing it we present formally some standard properties that will be also used.

The first axiom that we introduce is Anonymity. This property imposes that each agent's reward depends on the entire structure of the problem rather than her label.

Axiom 7. Anonymity ${ }^{5}$

Let $\varphi$ be a value for bankruptcy games. We say that $\varphi$ is anonymous if for each bankruptcy problem in $\mathcal{C}, B=(E, c)$, and any permutation $\pi$,

$$
\pi[\varphi(E, c)]=\varphi(E, \pi(c)) .
$$

The next axiom, called Continuity, requires that small changes in a bankruptcy problems does not cause big changes in the agents' rewards.

\footnotetext{
${ }^{5}$ The characterization result that we are going to provide is also true if Anonymity is weakened to Equal Tratment of Equals, that is, for each $(E, c) \in \mathcal{B}$ and each $\{i, j\} \subseteq N$, if $c_{i}=c_{j}$ then $\varphi_{i}(E, c)=$ $\varphi_{j}(E, c)$. We use Anonynity instead of Equal Treatment of Equals because we think that the adventages of doing so, concerning the simplification of the notation in the proofs, are fairly significant and moreover, Anonymity is a generally accepted property in our context, that is, bankruptcy problems with no priorities.
} 
Axiom 8. Continuity

Let $\varphi$ be a value for bankruptcy games. We say that $\varphi$ is continuous if for each sequence of bankruptcy problems in $\mathcal{C},\left\{\left(E^{n}, c^{n}\right)\right\}_{n=1, \ldots, \infty}$, that converges to a bankruptcy problem $(E, c) \in \mathcal{C}$, i.e. $\lim _{n \rightarrow \infty}\left(E^{n}, c^{n}\right)=(E, c)$,

$$
\varphi(E, c)=\lim _{n \rightarrow \infty} \varphi\left(E^{n}, c^{n}\right) .
$$

Remark 3. Note that Fixed Non-Quarrelled Claims Additivity and Continuity imply Invariance under Claims Truncation since given a bankruptcy problems in $\mathcal{C}, C=$ $(E, c)$, and a bankruptcy rule satisfying both properties, $\varphi$, we have that by Fixed Non-Quarrelled Claims Additivity,

$$
\begin{aligned}
\varphi(E, c)= & \varphi\left(E-(1 / r),\left[\min \left\{E, c_{i}\right\}\right]_{i \in N}-(1 / r)_{i \in N}\right)+ \\
& +\varphi\left((1 / r),\left[\max \left\{(1 / r), c_{i}-E+(1 / r)\right\}\right]_{i \in N}\right)
\end{aligned}
$$

for all $r$ such that

$$
r \in \mathbb{N},(1 / r)<\min \left\{\left\{c_{i}\right\}_{i \in N}, \max _{i \in N}\left\{c_{i}\right\}-E\right\} .
$$

Now, by considering the limit when $r$ goes to infinitum in the previous equation and taking into account that $\varphi$ is continuous we get

$$
\begin{aligned}
\varphi(E, c) & =\varphi\left(E,\left[\min \left\{E, c_{i}\right\}\right]_{i \in N}\right)+\varphi\left(0,\left[\max \left\{0, c_{i}-E\right]_{i \in N}\right)=\right. \\
& =\varphi\left(E,\left[\min \left\{E, c_{i}\right\}\right]_{i \in N}\right) .
\end{aligned}
$$

Theorem 6.3. Let $\varphi$ a bankruptcy rule, $\varphi$ satisfies Anonymity, Continuity and Fixed Non-Quarrelled Claims Additivity if, and only if, $\varphi \equiv \varphi^{m o}$.

Let us observe that the axioms used in Theorem 6.3 are independent. In particular, the Constrained Equal Awards bankruptcy rule is anonymous, continuous and does not satisfy Fixed Non-Quarrelled Additivity. The family of asymmetric Minimal Overlap bankruptcy rules, called Weighted Minimal Overlap Rules (see Alcalde, Marco and Silva [2] for a formal definition) satisfy Continuity and Fixed Non-Quarrelled Additivity and are not anonymous. Finally, the bankruptcy rule that recommends the Ibn Ezra's proposal for any bankruptcy problem in $\mathcal{C}, C=(E, c)$ whenever $\max _{i \in N}\left\{c_{i}\right\} \geq E$ and the Constrained Equal Losses otherwise, is anonymous, satisfies Fixed Non-Quarrelled Additivity but does not Continuity. 
Remark 4. To conclude this Section let us point out that the 'partial' Additivity property used to characterize the Minimal Overlap bankruptcy rule is no too exclusive. In fact, if we consider the domain of bankruptcy problems in which there is nobody demanding as much as there is, a very common situation, $(E, c) \in \mathcal{C}$ such that $\max _{i \in N}\left\{c_{i}\right\} \geq E$, the Constrained Equal Losses bankruptcy rule satisfies, in this domain, Fixed Non-Quarrelled Additivity.

\section{Conclusions}

This paper explored the possibility of finding additive bankruptcy rules. Following the (cooperative) game-theoretical approach of bankruptcy problems, and the close relationship they share with bargaining problems, we point out that there is a general impossibility of finding additive bankruptcy rules. This fact was shown by Bergantiños and Méndez-Naya [4].

We then explore the possibility of finding a restricted-additivity property that could be satisfied by bankruptcy rules. In this matter we find a positive result. When adding bankruptcy problems where agents share similar relative positions, not only among them but also relative to the estate, it is possible to find bankruptcy rules satisfying additivity. More than that, if we restrict to anonymous and continues rules, there is a unique possibility: the employ of the Minimal Overlap Rule proposed by O'Neill [10].

As a further research, which is already suggested in the paper, our approach gives an insight on how to extend the Perles and Mashler [13] solution to any set of agents. In fact, what it is somehow difficult, when adding bargaining problems, is to explore how individual's bargaining power is added. An interpretation, in terms of bargaining power, of our Fixed Non-Quarrelled Claims Additivity can be established as follows. If agents' bargaining power is similar in two problems, the selected bargaining solution should satisfy additivity in such a case. The question to be carefully explored is how to define similarity of agents' bargaining power.

\section{References}

[1] Alcalde, J., M.C. Marco and J.A. Silva (2005). 'Bankruptcy Games and the Ibn Ezra's Proposal', Economic Theory 26, 103-114.

[2] Alcalde, J., M.C. Marco and J.A. Silva (2008). 'The Minimal Overlap Rule Revisited', Social Choice and Welfare 31, 109-128.

[3] Aumann, R.J., and M. Maschler (1985). 'Game Theoretic Analysis of a Bankruptcy Problem from the Talmud', Journal of Economic Theory 36, 195-213. 
[4] Bergantiños, G. and L. Méndez-Naya (2001). 'Additivity in bankruptcy problems and in Allocation Problems'. Spanish Economic Review 3, 223-229.

[5] Calvo, E. and E. Gutiérrez (1994). 'Extension of the Perles-Maschler solution to n-person bargaining games', International Journal of Game Theory 23, 325-346.

[6] Chun, Y. and W. Thomson (2005). 'Convergence under Replication of Rules to Adjudicate Conflicting Claims', Games and Economic Behavior 50, 129-142.

[7] Curiel, I., M. Maschler and S. Tijs (1988). 'Bankruptcy Games', Z. Operations Research 31, 143-159.

[8] Dagan, N. and O. Volij (1993). 'The bankruptcy problem: a cooperative approach'. Mathematical Social Sciences 26, 287-297.

[9] Nash, J. F. (1950).'The Bargaining Problem'. Econometrica 28, 155-162.

[10] O'Neill, B. (1982). 'A Problem of Rights Arbitration from the Talmud', Mathematical Social Sciences 2, 345-371.

[11] Palaschke, D. and J. Rosenmüller (2007). 'A superadditive solution for cephoidal bargaining problems', International Journal of Game Theory 35, 569-590.

[12] Perles, M. A. (1982). 'Non-existence of superadditive solution for 3-person games', International Journal of Game Theory 11, 151-161.

[13] Perles, M. A. and M. Maschler (1981) 'The Super-Additive Solution for the Nash Bargaining Game', International Journal of Game Theory 10, 163-193.

[14] Peters, H. (1986). 'Simultaneity of Issues and Additivity in Bargaining'. Econometrica 54, 153-169.

[15] Shapley, L.S. (1953). 'A Value for N-person Games'. In Contributions to the Theory of Games II, H.W. Karlin and A.W. Tucker eds. Princeton University Press.

[16] Thomson, W. (2003). 'Axiomatic and game-theoretic analysis of bankruptcy and taxation problems: a survey', Mathematical Social Sciences 45, 249-297.

[17] Thomson, W. (1994). 'Cooperative Models of Bargaining' In Handbook of Game Theory with Economics Applications, Volume 2: 1237-1284. (R. Aumann and S. Hart eds.) North Holland.

[18] Thomson, W. (2009). Bargaining Theory: The Axiomatic Approach. Academic Press, forthcoming 
[19] Von Neumann, J. and O. Morgenstern (1944). Theory of Games and Economic Behavior. Princeton: Princeton University Press; 2nd edn. 1947; 3rd edn. 1953. 


\section{Appendix}

This appendix provides a formal proof for Theorem 6.3.

\section{Proof of Theorem 6.3.}

First, it is straightforward to check that the Minimal Overlap Rule satisfies Anonymity, Continuity and Fixed Non-Quarrelled Claims Additivity.

Now, let $\varphi$ be a rule satisfying these axioms. Let us consider a problem $(E, c) \in \mathcal{B}$. By Anonymity we can assume, w.l.o.g., that $c$ is increasingly ordered i.e., $c_{i} \leq c_{j}$ whenever $i<j$.

Let us consider the following two cases:

Case [1] $E \leq c_{n}$.

By Fixed Non-Quarrelled Claims Additivity and Continuity (See Remark 3) we have that

$$
\varphi(E, c)=\varphi(E, \tilde{c})
$$

where the $i$-th component of $\tilde{c}$, the vector of truncated claims, is

$$
\tilde{c}_{i}=\min \left\{c_{i}, E\right\} .
$$

Let us denote $P^{1}=\tilde{c}_{1}$, for $1<i \leq n, P^{i}=\tilde{c}_{i}-\tilde{c}_{i-1}$ and for all $i \in N$, let denote $c_{k}^{P^{i}}=\max \left\{P^{i}, 0\right\}$.

Now, let us consider the following two subcases:

$[1]-(a) \tilde{c}_{n}=\tilde{c}_{n-1}$.

From Fixed Non-Quarrelled Claims Additivity we get that

$$
\varphi(E, \tilde{c})=\sum_{i / P^{i} \neq 0} \varphi\left(P^{i}, c^{P^{i}}\right) .
$$

Now, let us observe that, for each $i$, we have that, if $P^{i}>0$ then $c_{j}^{P^{i}}=0$ for all $j<i$, and $c_{j}^{P^{i}}=c_{i}^{P^{i}}$ for each $j \geq i$. Therefore, by Anonymity

$$
\varphi_{j}\left(P^{i}, c^{P^{i}}\right)=\left\{\begin{array}{cc}
0 & \text { if } j<i \\
\frac{P^{i}}{n-i+1} & \text { if } j \geq i
\end{array},\right.
$$

i.e.

$$
\varphi_{j}\left(P^{i}, c^{P^{i}}\right)=\left\{\begin{array}{cc}
0 & \text { if } j<i \\
\frac{\tilde{c}_{i}-\tilde{c}_{i-1}}{n-i+1} & \text { if } j \geq i
\end{array}\right.
$$


with $c_{0}=0 .^{6}$ And, thus, for each agent $h$

$$
\begin{aligned}
\varphi_{h}(E, \tilde{c}) & =\sum_{i / P^{i} \neq 0} \varphi_{h}\left(P^{i}, c^{P^{i}}\right)=\sum_{i \leq h / P^{i} \neq 0} \varphi_{h}\left(P^{i}, c^{P^{i}}\right)= \\
& =\sum_{i \leq h / P^{i} \neq 0} \frac{\min \left\{c_{i}, E\right\}-\min \left\{c_{i-1}, E\right\}}{n-i+1}= \\
& =\sum_{i=1}^{h} \frac{\min \left\{c_{i}, E\right\}-\min \left\{c_{i-1}, E\right\}}{n-i+1}=\varphi_{h}^{m o}(E, c) .
\end{aligned}
$$

$[1]-(b) \tilde{c}_{n} \neq \tilde{c}_{n-1}$.

Let us denote by $q(j)$ the cardinal of the set $\left\{P^{i}, i \leq j\right.$, such that $\left.P^{i} \neq 0\right\}$. From Fixed Non-Quarrelled Claims Additivity we get that

$$
\begin{aligned}
\varphi(E, \tilde{c})= & \varphi\left(P^{1}+\alpha,\left(\left\{c^{P^{1}}\right\}_{i<n}, c^{P^{1}}+\alpha\right)+\right. \\
& +\sum_{1<i<n / P^{i} \neq 0} \varphi\left(P^{i}-(\alpha /[q(n)-1]),\left\{\max \left\{0, c^{P^{i}}-(\alpha /[q(j)-1])_{j \in N}\right\}\right)+\right. \\
& +\varphi\left(P^{n}-[\alpha /[q(n)-1]],\left(0,\{\alpha\}_{1<i<n}, c_{n}^{P^{n}}-[\alpha /[q(n)-1]]\right),\right.
\end{aligned}
$$

where

$$
\alpha=(1 / r), r \in \mathbb{N}, \alpha<\min \left\{\left\{P^{i}\right\}_{P^{i} \neq 0}, P^{n}([q(n)-1] / q(n))\right\} .
$$

Now, by considering the limit when $r$ goes to infinitum in the previous equation and taking into account that $\varphi$ is continuous we get

$$
\varphi(E, \tilde{c})=\sum_{i / P^{i} \neq 0} \varphi\left(P^{i}, c^{P^{i}}\right) .
$$

And from now on, by using the reasoning of the subcase [1] - (a) we get that for each agent $h$

$$
\varphi_{h}(E, \tilde{c})=\varphi_{h}^{m o}(E, c)
$$

Case [2] $E>c_{n}$.

In such a case, there is a unique $t, 0 \leq t<c_{n}$ such that $\sum_{j=1}^{n} \max \left\{0, c_{j}-t\right\}=E-t$. Let $k$ be the unique agent such that $c_{k}-t>0$, and $c_{k-1}-t \leq 0$. So that, the claim of the $j$-th agent is partially indisputable in $(E, c)$ only for all $j \geq k$.

\footnotetext{
${ }^{6}$ Throughout this proof, and for notational convenience, we will consider $c_{0}=0$.
} 
Then, by Fixed Non-Quarrelled Claims Additivity we have that

$$
\begin{aligned}
\varphi(E, c)= & \varphi\left(t+\alpha,\left(\left\{c_{i}\right\}_{i<k},\left\{t+(\alpha /(n-k+1)\}_{i \geq k}\right)+\right.\right. \\
& +\varphi\left(E-t-\alpha,\left(\{0\}_{i<k},\left\{c_{i}-t-(\alpha /(n-k+1)\}_{i \geq k}\right)\right),\right.
\end{aligned}
$$

where

$$
\alpha=(1 / r), r \in \mathbb{N}, \alpha<\min \left\{E-t,(n-k+1)\left(c_{k}-t\right)\right\} .
$$

Now, by considering the limit when $r$ goes to infinitum in the equation [7.1]and taking into account that $\varphi$ is continuous we get

$$
\varphi(E, c)=\varphi\left(t,\left[\min \left\{c_{i}, t\right\}\right]_{i \in N}\right)+\varphi\left(E-t,\left[\max \left\{0, c_{i}-t\right\}\right]_{i \in N}\right) .
$$

Let us observe that the problem $\left(t,\left[\min \left\{t, c_{i}\right\}\right]_{i \in N}\right)$ was analyzed in case $[1]$ above. Therefore, for each agent $h$, we have that

$$
\varphi_{h}\left(t,\left[\min \left\{t, c_{i}\right\}\right]_{i \in N}\right)=\sum_{i=1}^{h} \frac{\min \left\{c_{i}, t\right\}-\min \left\{c_{i-1}, t\right\}}{n-i+1} .
$$

Moreover, note that for agent $h$ we have that

$$
\max \left\{0, c_{h}-t\right\}=\left\{\begin{array}{cc}
0 & \text { if } h<k \\
c_{h}-t & \text { if } h \geq k
\end{array} .\right.
$$

Since by construction

$$
\sum_{i=1}^{n} \max \left\{0, c_{i}-t\right\}=E-t
$$

we can conclude

$$
\varphi\left(E-t,\left[\max \left\{0, c_{i}-t\right\}\right]_{i \in N}\right)=\left[\max \left\{0, c_{i}-t\right\}\right]_{i \in N} .
$$

Therefore, by combining equations [7.1], [7.2], and [7.3], we have that, for each agent $h$,

$$
\varphi_{h}(E, c)=\sum_{i=1}^{h} \frac{\min \left\{c_{i}, t\right\}-\min \left\{c_{i-1}, t\right\}}{n-i+1}+\max \left\{0, c_{h}-t\right\}=\varphi_{h}^{m o}(E, c) .
$$

